Recepción: 10/01/2019

Aceptación: 08/02/2019

Publicación: 05/04/2019

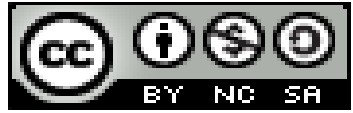

Ciencias económicas y empresariales

Artículos de revisión

\title{
La cultura financiera y la creación de emprendimientos en la ciudad de Loja- Ecuador
}

The financial culture and the creation of enterprises in the city of Loja-Ecuador

\author{
A cultura financeira e a criação de empresas na cidade de Loja-Equador
}

\author{
Mary Beatriz Maldonado-Román ${ }^{\mathrm{I}}$ \\ m.a.beatriz@hotmail.com \\ Verónica Cecilia Cabrera-González II \\ veronays@hotmail.com \\ Mariuxi Cecibel Duarte-Torres ${ }^{\text {III }}$ \\ marydt18@hotmail.com \\ Mirian Esperanza Rodríguez-Calva ${ }^{\text {IV }}$ \\ mariesperanza1979@gmail.com
}

Correspondencia: m.a.beatriz@hotmail.com

\footnotetext{
I Magíster en Gerencia Contable y Financiera, Doctora en Contabilidad y Auditoria, Licenciado en Contabilidad y Auditoría-Contador Público Auditor, Carrera de Contabilidad y Auditoría, Docente Universidad Nacional de Loja, Loja, Ecuador.

${ }^{\text {II }}$ Magíster en Finanzas, Licenciada en Contabilidad y Auditoría, Contador Público-Auditor, Carrera de Contabilidad y Auditoría, Docente Universidad Nacional de Loja, Loja, Ecuador.

III Magíster en Auditoria Integral, Licenciada en Contabilidad y Auditoría Contador Público Auditor, Carrera de Contabilidad y Auditoría, Docente Universidad Nacional de Loja, Loja, Ecuador.

IV Magíster en Gestión Empresarial, Licenciada en Contabilidad y Auditoría, Contador Público-Auditor, Carrera de Contabilidad y Auditoría, Docente Universidad Nacional de Loja, Loja, Ecuador.
} 


\section{Resumen}

Las Mipymes en el contexto mundial, constituyen la fuerza motriz del crecimiento económico, en Latinoamérica y los países de la región, su lugar en la economía reviste gran importancia debido a que se estima que el 95\% del total de empresas corresponden a este tipo de emprendimientos. Según el último Censo Nacional Económico del 2010, en el Ecuador aproximadamente 99 de cada 100 establecimientos se encuentran dentro de la categoría de MIPYMES (INEC, 2010). Esta particularidad se convierte en un dato clave sobre el valor que tiene este sector a la hora de aportar al proceso de consolidación del sistema productivo nacional. Tomando en cuenta este referente, se desarrolló un análisis con la finalidad de identificar las limitaciones que tienen los emprendimientos para acceder al microcrédito como fuente de financiamiento. Se tomó como referente estudios de investigaciones realizadas y se consultó datos estadísticos sobre el tema, lo que permitió obtener como resultado la identificación de las limitaciones, así como determinar el rol de la inclusión financiera para el desarrolla de la actividad emprendedora. Concluyendo que en el país se implementaron políticas públicas para apoyar preferentemente al pequeño emprendedor, con un marco legal que ha determinado tasas de interés bajas denominados créditos vip, se logró estandarizar el papeleo y la banca pública formo parte de este proceso. En la actualidad existe la percepción de que todo este trabajo se ha estancado debido a la inacción del gobierno en el fomento de alternativas viables para apoyar el emprendedor ecuatoriano.

Palabras Clave: volatilidad financiera; inclusión financiera; contracción económica; inserción productiva; esfera macroeconómica.

\section{Abstract}

Mipyme in the global context, constitute the driving force of economic growth, in Latin America and the countries of the region, its place in the economy is of great importance because it is estimated that $95 \%$ of all companies correspond to this type of ventures. According to the last National Economic Census of 2010, in Ecuador approximately 99 out of 100 establishments are in the category of MSMEs (INEC, 2010). This particularity becomes a key fact about the value that this sector has when contributing to the consolidation process of the national productive system. Taking into account this referent, an analysis was developed with the purpose of identifying the limitations that entrepreneurs have to access microcredit as a source of financing. Reference was made to research studies and statistical data on the subject were consulted, which

\section{6}

Pol. Con. (Edición núm. 32) Vol. 4, No 4, marzo 2019, pp. 105-125, ISSN: 2550 - 682X 
allowed obtaining as a result the identification of the limitations, as well as determining the role of financial inclusion for the development of the entrepreneurial initiative. In the country, public policies were implemented to support the small entrepreneur, with a legal framework that has determined low interest rates called vip loans, standardization of paperwork and public banking was part of this process. At present, there is a perception that all this work has stalled due to the inaction of the government in the promotion of viable alternatives to support the Ecuadorian entrepreneur.

Keywords: Financial volatility, financial inclusion, economic contraction, productive insertion, macroeconomic sphere.

\section{Resumo}

As MPMEs no contexto global, constituem a força motriz do crescimento econômico, na América Latina e nos países da região, seu lugar na economia é de grande importância, pois estima-se que $95 \%$ de todas as empresas correspondam a esse tipo de empreendimentos. De acordo com o último Censo Econômico Nacional de 2010, no Equador, aproximadamente 99 de 100 estabelecimentos estão na categoria de MPMEs (INEC, 2010). Essa particularidade torna-se um fato fundamental sobre o valor que esse setor tem ao contribuir para o processo de consolidação do sistema produtivo nacional. Tendo em conta este referente, foi desenvolvida uma análise com o objetivo de identificar as limitações que os empreendedores têm para acessar o microcrédito como fonte de financiamento. Foi tomado como uma pesquisa de referência e dados estatísticos foram consultados sobre o assunto, o que resultou na identificação das limitações, bem como na determinação do papel da inclusão financeira para o desenvolvimento da atividade empreendedora. Concluindo que no país foram implementadas políticas públicas para apoiar preferencialmente o pequeno empreendedor, com um arcabouço legal que determinou baixas taxas de juros denominadas empréstimos VIP, foi possível padronizar a papelada e os bancos públicos fizeram parte desse processo. Atualmente, há uma percepção de que todo esse trabalho foi interrompido devido à inação do governo na promoção de alternativas viáveis para apoiar o empreendedor equatoriano.

Palavras-chave: volatilidade financeira; inclusão financeira; contração econômica; inserção produtiva; esfera macroeconômica. 


\section{Introducción}

Los retos en cuanto a política fiscal y las reformas monetarias que enfrentan los países, frente a la volatilidad financiera y la incertidumbre económica a nivel global han disminuido las perspectivas de la dinámica de crecimiento en los países de Latinoamérica.

En el año 2016 según la (Perspectivas Económicas de America Latina 2017 OCDE/Naciones Unidas/CAF, 2016) el PIB decayó en forma notable en América Latina, produciendo una contracción económica que venía ya desde el 2015.

Frente a esto, los países latinoamericanos exhiben ralentización en la economía, enfrentando diversos problemas de liquidez, lo que los ha llevado a delinear políticas públicas que permiten la inclusión financiera a organizaciones y entidades que anteriormente no tenían acceso al sistema, del mismo modo se apunta al perfeccionamiento de la utilización del sistema financiero por parte de las que participan en el entorno financiero formal. (Jim Yong Kin, 2015) En este proyecto, se considera que las "políticas públicas y las medidas de inclusión financiera de las instituciones financieras de desarrollo son iniciativas que apuntan a la inserción productiva de las empresas de menor tamaño, mediante el fortalecimiento de su capacidad y la ampliación de posibilidades de inversión y, en virtud de la bancarización, permitir que los individuos y las empresas se integren a la esfera de la producción y el consumo" (João Carlos Ferraz \& Luma Ramos, 2018)

En este contexto en pleno siglo XXI, la apertura de negocios pequeños que ingresa en la esfera macroeconómica se ha incrementado, son muchos los individuos con iniciativas e ideas para iniciar un negocio propio que no tienen una cultura financiera, carecen de conocimiento en cuanto al ahorro, fuentes de financiamiento, como manejar los créditos, etc.

Por ello es importante que exista una adecuada educación financiera que les permita hacer que su dinero trabaje para obtener rentabilidad, que pueda capitalizarla en favor de su negocio y a la vez diversificarlo en beneficio de los consumidores y el suyo propio.

En el presente artículo se aborda el tema de la educación financiera como el pilar fundamental para el desarrollo de los pequeños negocios implementados por los llamados emprendedores, individuos con visión, ideas brillantes, pero con escasa educación en el manejo económico y

\section{8}

Pol. Con. (Edición núm. 32) Vol. 4, No 4, marzo 2019, pp. 105-125, ISSN: 2550 - 682X 
financiero (Romero , 2017), así como el rol que cumple la inclusión financiera para la promoción del emprendimiento.

En el contexto Latinoamericano, Ecuador es el país donde más negocios de este tipo se han creado (Zamora Boza C. S., 2018), ello va de la mano con el fortalecimiento de políticas públicas, que durante estos últimos 10 años han sido un política prioritaria en el país, él apoyo al micro y pequeño empresario ha sido acompañado con el establecimiento de un marco legal, normad, programas, proyectos, instituciones, que permitan afianzar las nuevas ideas y sembrar las bases para el fomento de los emprendimientos, promoviendo el dinamismo de este sector coadyuvando al desarrollo socioeconómico del país.

\section{Metodología}

El desarrollo del artículo se enmarca en un análisis descriptivo de las variables que intervienen en la creación de emprendimientos en la ciudad de Loja-Ecuador, particularmente, la educación y cultura financiera, por medio del análisis de las diversas fuentes bibliográficas que permitieron su concepción, se inicia con el estudio del marco conceptual de la educación financiera, determinando el rol que cumplen la inclusión financiera en la promoción del emprendimiento, se identifican las limitaciones que tienen los emprendimientos para acceder al microcrédito como fuente de financiamiento,. Finalmente se detallan las variables que deben tenerse en cuenta para formar individuos emprendedores

\section{Desarrollo}

La educación financiera se define como: "el proceso por el cual los consumidores, inversionistas financieros, mejoran su comprensión de los productos financieros, los conceptos y los riesgos, y, a través de información, instrucción y/o el asesoramiento objetivo, desarrollan las habilidades y confianza para ser más conscientes de los riesgos y oportunidades financieras, tomar decisiones informadas, saber a dónde ir para obtener ayuda y ejercer cualquier acción eficaz para mejorar su bienestar económico" (Serie Políticas Públicas y Transformación Productiva Nº12, 2013).

(Yurani Ardila \& Rengifo Ariza, Aproximaciones a la Educación Financiera en América Latina, 2014) Considerando el poco interés por la cultura financiera en la población, sugiere la necesidad de incentivar el conocimiento y desarrollo de estas capacidades, mediante el establecimiento de políticas de educación financiera como una alternativa que permita promover cambios positivos 
en el comportamiento económico y en los niveles de educación financiera de las personas y los hogares.

(Dominguez Martinez J. M., 2017) el poco interés de una educación financiera trae consigo muchas dificultades, tomando en cuenta que en América Latina el poco acceso a los servicios financieros es limitado, la realidad es que las grandes instituciones financieras ponen demasiadas trabas para el acceso a fuentes de financiamiento.

Es necesario hablar de la inclusión financiera como el proceso de promoción de un camino factible, oportuno y adecuado a una amplia gama de productos y servicios financieros regulados y la ampliación de su uso por todos los segmentos de la sociedad, a través de la aplicación de enfoques innovadores o existentes, incluyendo actividades de sensibilización y de educación financiera, con el fin de promover el bienestar económico y la inclusión económica y social.

Debido a los cambios vertiginosos que se han dado en el presente siglo, aunado al surgimiento de mercados financieros cada vez más sofisticados, los cuales han contribuido a la formación de esta conciencia cada vez mayor, especialmente en los individuos que tienen la iniciativa de emprender en negocios pequeños. Según (Espín Oleas, Loza Montes, \& Castillo Armijos, Educación Financiera, clave para reducir la pobreza y la desigualdad, 2018) por la década de los sesenta se da inicio a los primeros programas de educación financiera en los países desarrollos principalmente en Estados Unidos, inicialmente estos programas presentaron algunas debilidades, sin embargo como una posible medida de solución se incluye al individuo para que forme parte de los programas de educación financiera y de esta manera poder mejorar las capacidades de los individuos, siendo este interés no únicamente de las instituciones públicas y privadas de Estados Unidos sino de todo el mundo.

La inclusión financiera en América Latina, se la considera, según (Roa, Inclusión financiera en América Latina y el Caribe; acceso, uso y calidad, 2013) de suma importancia porque constituye una fuente que permite contrarrestar la desigualdad social y la pobreza. Es importante señalar que se debe regular las entidades que se dedican a la intermediación financiera como medios responsables de esta inclusión, será necesario adjuntar la regulación, supervisión de los diferentes

\section{0}

Pol. Con. (Edición núm. 32) Vol. 4, No 4, marzo 2019, pp. 105-125, ISSN: 2550 - 682X 
productos y servicios financieros que ofrecen, etc. Es decir, es ineludible que estas instituciones se conviertan en la vía para una educación financiera básica y eficaz.

Es por ello que la Inclusión financiera según (Espín Oleas, Loza Montes, \& Castillo Armijos, Educación Financiera, clave para reducir la pobreza y la desigualdad, 2018) conlleva tener actitud positiva, superación, empoderamiento, lo que servirá de aprendizaje en todas las etapas de la vida. Una educación financiera empieza desde una temprana edad: niño, adolescente adulto; inculcando en la persona el valor del dinero y el entendimiento de un presupuesto, que se fundamente en las premisas de cómo ahorrar, como trabajar; como invertir, hacer que el dinero genere una rentabilidad y así enfocarnos a un emprendimiento a futuro.

(Guerreo Jaimes, Villamizar Ramírez, \& Maestre Delgado, 2018) Cuando se habla de ahorrar, el enfoque se direcciona a las finanzas personales, la mayoría de la población no maneja esta cultura, sin embargo, lo correcto sería destinar el $10 \%$ de los ingresos que se percibe al ahorro, pero en la realidad ésta práctica no se aplica. Ahorrar implica la seguridad de cumplir con los objetivos o metas a corto y largo plazo (Villada, López Lezama, \& Muñoz Galeano, 2017).

Los programas de educación financiera e inclusión no solamente se basan en impartir conocimientos, si no enfocarse en las actitudes y principios de la importancia del ahorro. La capacidad de ahorrar se basa en mecanismos formales como: vivir en una zona urbana, contar con empleo seguro a tiempo completo y el nivel educativo, según (Mejía Anzola \& Rodriguez Guzmán, Determinantes socioeconómicos de la Educación Financiera. Evidencia para Bolivia, Colombia, Ecuador y Perú, 2016)

El objetivo de la inclusión financiera es facilitar el camino al mercado globalizado de la población que no necesariamente tiene acceso a los productos y servicios financieros que el mercado ofrece, según (Moncayo \& Reis, 2016).

\section{Aspectos de la falta de Educación Financiera}

Según (Amezcua García, Arroyo Grant, \& Espinoza Mejia, Contexto de la Educación Financiera en México, 2014) los datos de la falta de educación financiera, lo interpreta de la siguiente manera: 
- Poca participación en los cursos que ofrecen las entidades financieras de los grupos sociales, sectores de la sociedad civil, etc.

- El desconocimiento de los diferentes productos y servicios financieros que ofrece la banca.

- Falta de empoderamiento de los derechos y obligaciones como clientes.

- Falta de una planificación financiera.

En el Ecuador la población más vulnerable se enfrenta a barreras como la distancia, la ausencia de garantías y, sobre todo, la falta de confianza de los proveedores de servicios financieros. La exclusión financiera se concentra en determinados perfiles de población como: mujeres, indígenas y población rural alejada.

La implementación de políticas de Estado ha mejorado la situación de quienes buscan acceso a este tipo de información, ello se refleja en la constitución de la República concretamente en el art. 52, que determina "las personas tienen derecho a disponer de bienes y servicios de óptima calidad, y a elegirlos con libertad; así como a una información precisa y no engañosa sobre su contenido y características". La Superintendencia de Bancos acogiéndose a lo que manda la constitución mediante resolución SB-2015-665, dispone que las entidades financieras desarrollen programas de Educación Financiera a favor de sus clientes y usuarios en general con el propósito de apoyar a la formación de conocimientos en este campo. (Banco del Estado Ecuador, 2015)

\section{Emprendimientos}

Un negocio nace de una idea que, por muchas razones, mueve en una o más personas el interés suficiente para comenzar en un arduo e incierto viaje que tiene por objetivo convertir en realidad dicha idea (Fundación Suiza en Bolivia, 2017).

(Romero Martinez \& Milone, 2016) Los emprendedores son los principales creadores de empleo facilitando la regeneración económica y social de los países. La situación de crisis económicafinanciera y social acontecida en los últimos años en algunos países, en especial España ha dado lugar a un nuevo escenario que suscita especialmente la necesidad de profundizar en el estudio de los determinantes del emprendimiento, Cada persona decide emprender por determinados motivos y aunque su decisión es personal, lo cierto que en la mayoría de los emprendedores se

\section{2}

Pol. Con. (Edición núm. 32) Vol. 4, No 4, marzo 2019, pp. 105-125, ISSN: 2550 - 682X 
valora algunas ventajas frente a un trabajador en relación de dependencia, el poder desarrollar tus propias ideas, ser el dueño de tu carrera profesional, tener libertad, entre otras.

(Lederman, Messina, Pienknagura, \& Rigolini, 2014) El emprendimiento es un motor de crecimiento, es por ello que los emprendedores de éxito son individuos que transforman ideas en iniciativas rentables. A menudo, esta transformación requiere talentos especiales, como la capacidad de innovar, introducir nuevos productos y explorar otros mercados. Se trata de un proceso que también precisa la habilidad de dirigir a otras personas, priorizar las tareas para aumentar la eficiencia productiva y darles a los recursos disponibles el mejor uso posible. Sin embargo, no basta con estas aptitudes, exige de esfuerzo, trabajo, voluntad, acompañado de una actitud proactiva y positiva.

Particularmente, según (Zamora Boza C. , 2018) el 19\% de los jóvenes latinoamericanos se encuentran dentro de un empleo informal, mientras que el $20 \%$ no estudia ni trabaja (OCDE/CEPAL/CAF, 2016). Bajo este panorama, el emprendimiento tiene un papel clave en el desarrollo del auto empleado. Un independiente creativo que cuente con las motivaciones, capacidades e instrumentos necesarios, pasa de auto empleado a emprendedor y desempeña actividades productivas de mayor valor, produce más, genera más ganancias y contrata a más empleados, esto permite la creación de más y mejor empleo.

El emprendimiento suele ser un proyecto que se desarrolla con esfuerzo y haciendo frente a diversas dificultades, con la resolución de llegar a un determinado punto. En este caso, un emprendimiento es una iniciativa de un individuo que asume un riesgo económico o que invierte recursos con el objetivo de aprovechar una oportunidad que brinda el mercado. La persona que inicia un negocio o que crea una pequeña empresa por su propia iniciativa se conoce como emprendedor, los emprendedores deben contar con ciertas capacidades para tener éxito: flexibilidad, dinamismo, creatividad, empuje, etc. Se trata de valores necesarios ya que los emprendimientos se enfrentan a todo tipo de dificultades y quien los impulsa debe estar en condiciones de adaptarse a una realidad cambiante.

(Moreno Zacarías \& Olmos , 2010) En un estudio realizado en la ciudad de México en 2016, puntualizan; que el desarrollo de las habilidades de los emprendedores que conviertan sus negocios en empresas de éxito, obedece en su mayoría al sistema de la "triple hélice" la cual 
hace referencia a la relación universidad-gobierno-empresa, las actividades emprendedoras tienen mucho que ver con la capacidad de liderazgo para la gestión de dichos negocios, tomando como referencia el análisis de varios países de Asia (Japón, Malasia y Filipinas) y Latinoamérica (México, Brasil, Argentina y Chile), se evidencia que en Asia el desarrollo de emprendedores se enfocan a la innovación de las empresas, no así en los países de Latinoamérica en donde se constituyen emprendedores por necesidad y con el afán de buscar una opción de trabajo que les permita suplir sus necesidades de consumo.

\section{Resultados}

\section{Inclusión Financiera}

\section{Grafico 1 Encuesta: Informe de Inclusión Financiera 2016 Ecuador}

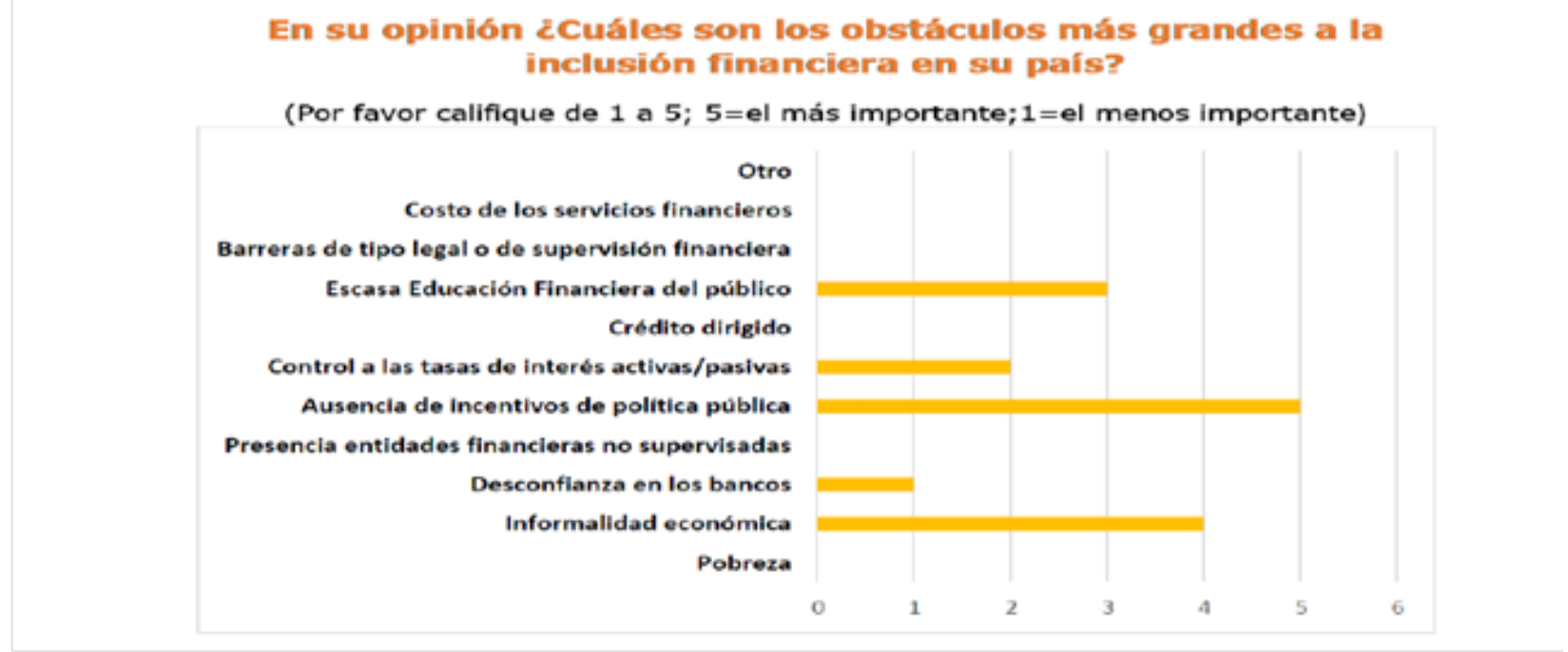

Fuente: (Feleban, 2016)

En el grafico No. 1 se expone los principales obstáculos que limitan una mayor inclusión financiera en el Ecuador, tales como: la ausencia de incentivos de política pública, informalidad económica, y la escasa educación financiera del público (FELABAN, 2016) según el informe de Inclusión Financiera el mayor desafío es generar en el público la confianza hacia la banca, resultado que beneficiaria positivamente a las instituciones financieras ya que les permitirá captar nuevos clientes para ofertar sus productos y servicios; y, en la población fomentar una

\section{4}

Pol. Con. (Edición núm. 32) Vol. 4, No 4, marzo 2019, pp. 105-125, ISSN: 2550 - 682X 
cultura de ahorro además de la posibilidad de desarrollar sus ideas de negocio con el financiamiento y de esta manera generar crecimiento económico y empleo.

Así mismo (Fernández de Lis \& Pacheco , 2017) describen algunos factores que podrían afectar la inclusión financiera tales como: factores socioeconómicos (ingresos, desigualdad, barreras, desarrollo social), macroeconómicos (riqueza de la economía o estabilidad del sistema financiero), institucionales (informalidad o fallas del control social, leyes débiles) factores relacionados con el sistema financiero (eficiencia del sector bancario, elevada concentración).

Según datos expuestos en el informe de la (Red de Instituciones Financieras de Desarrollo, 2017) en el Ecuador el $46 \%$ de los adultos tienen acceso a una cuenta bancaria y únicamente el 23\% de ellos tomo un crédito en el 2014, estos indicadores nos demuestran que Ecuador en todas las variables de acceso a crédito (por edad, género y pobreza) está por debajo del promedio en Latinoamérica. Con lo mencionado se denota que existe un potencial grupo excluido aun por atender, en este informe la Red de Instituciones Financieras de Desarrollo (RDF), propone la construcción de una política nacional de inclusión financiera y de desarrollo socioeconómico para este sector excluido, dicha política incluye el acceso y uso de servicios financieros, regulación apropiada, protección al usuario financiero y educación financiera.

Describe la carrera empresarial y la educación financiera como factores esenciales, donde se complementan la motivación y la formación académica para la creación de empresas, las capacitaciones, cursos, asesoramiento técnico etc. lo que conlleva a la formación de un emprendedor de éxito. El conocimiento empodera y genera ventaja competitiva además que minimiza los riesgos al fracaso y potencializa el éxito esperado del emprendedor. (García, Griofoni, López, \& Mejía, 2013), en su estudio concluye diciendo que la educación financiera se ha convertido en una prioridad para las instituciones públicas a nivel mundial.

Se realizó un diagnóstico con la finalidad de identificar las fuentes de financiamiento de las Mipymes en los países de América Latina, donde según (Saavedra Garcia \& León Vite, 2014) obteniéndose como resultado que la principal fuente de financiamiento para la micro, pequeña y mediana empresa, son los recursos propios y la mayor limitante para no solicitar un crédito en el sector financiero son las elevadas tasas de interés. Lo que denota que las políticas implementadas en estos países no han cumplido completamente su propósito, por lo que es necesario que se tomen acciones eficientes que permitan apoyar y fortalecer el sector empresarial en beneficio de 
dinamizar la economía de los países latinoamericano, creándose líneas de créditos específicas para atender los diversos destinos de inversión, periodos de gracias para quienes van a emprender, plazos en relación a la recuperación de la inversión, formas de pago en función a los flujos de efectivo de sus negocios, la opción de presentar garantías quirografarias o reales, así como la simplificación en la documentación requerida para ser sujeto o acreedor a un financiamiento, inclusive sin historial crediticio. Con ello las empresas podrán optar a un crédito de acuerdo a sus necesidades y operar con capital suficiente para asegurar un crecimiento adecuado y sostenido, que les permita permanecer en el mercado.

En Ecuador con el lema "todo el mundo mejor" se institucionaliza el (Plan Nacional para el Buen Vivir , 2017-2021), concebido como instrumento político que marca la hoja de ruta técnica que direcciona el accionar del sector público, que busca cumplir con el mandato de "planificar el desarrollo nacional, erradicar la pobreza, promover el desarrollo sustentable y la redistribución equitativa de los recursos y la riqueza, para acceder al buen vivir" a partir de ello se establecen objetivos, lineamientos y metas para los tres ejes señalados y los nueve objetivos nacionales. (Salas, Espinoza, \& Samaniego, 2016) se refiere al Plan Nacional del Buen Vivir, como un nuevo modelo económico que da gran prioridad a los procesos productivos del sector popular y solidario, determina el potencial de las micro-finanzas en el empoderamiento de las personas emprendedoras.

(Zúñiga Santillán, Espinoza Toalombo, Campos Rocafuerte, Tapia Núñez, \& Muñoz Bernal, 2016) Las Mipymes en Ecuador son fundamentales en la economía, se han convertido en una fuente de generación de empleo y un medio para ofrecer productos y servicios diversos en mercados de menor tamaño. El apoyo del Estado, se evidencia en la contribución con organismos estatales como: MICIP (Ministerio de Industrias y Competitividad), La Subsecretaría de la Micro, Pequeñas y Medianas Industrias y Artesanías, la CAF (Corporación Financiera Nacional), etc., esta intervención del estado está permitiendo el desarrollo y competitividad de las Mipymes, especialmente en las siguientes temáticas de negocios: capacitación de recursos humanos, asistencia técnica, asociatividad empresarial, promoción de exportaciones y compras públicas como comercialización interna.

\section{6}

Pol. Con. (Edición núm. 32) Vol. 4, No 4, marzo 2019, pp. 105-125, ISSN: 2550 - 682X 
En el Ecuador las Mipymes se han venido desarrollando en el campo de la producción de bienes y servicios constituyéndose en el pilar fundamental del desarrollo productivo del país, muchas de estas empresas se han conformado con los créditos otorgados de las instituciones financieras. Sin embargo, algunas de ellas han tenido un ciclo de vida corto ya que no cumplen con los requerimientos dela banca para la concesión de los préstamos, así como su evolución se vuelve lenta al no contar con las innovaciones tecnológicas que les permitan crecer ya que lo ven como un gasto y no como una inversión recuperable en el tiempo. Pese a estos escenarios adversos las Pymes en el Ecuador representan el 95\% de las unidades productivas y el 60\% del empleo del país (Delgado Delgado \& Chávez Granizo, 2018)

\section{Grafico 2}

\section{Tamaño medio de las empresas y nivel de desarrollo (promedio 2004-2008)}

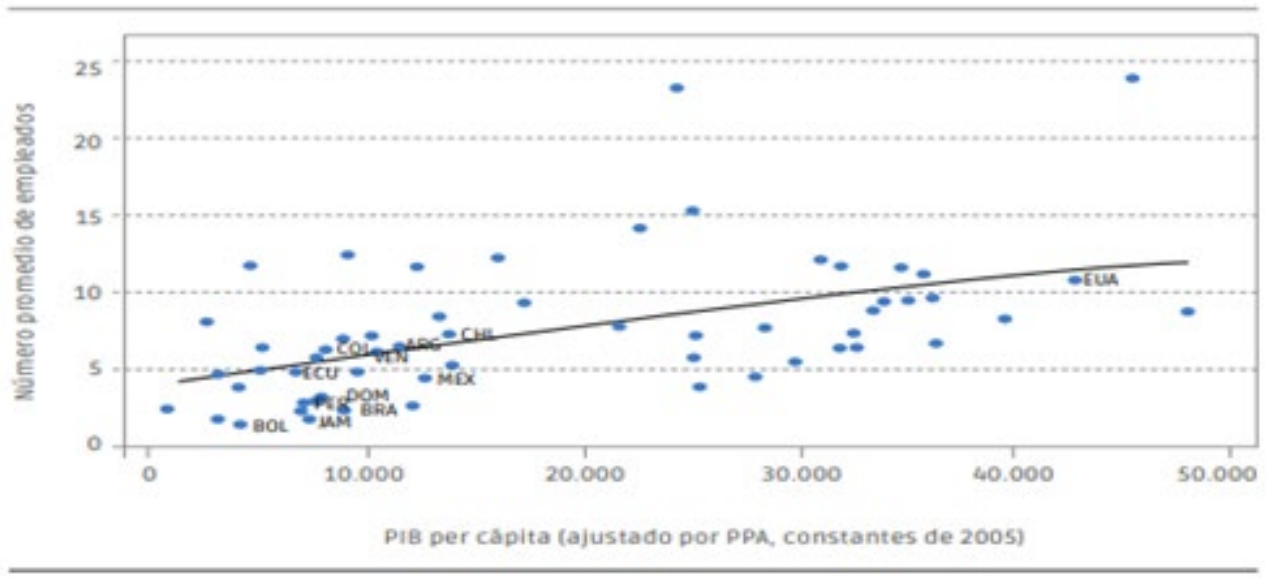

Fuente: elaboracuon propia con base de GEM (2012)

Según, (Lederman, Messina, Pienknagura, \& Rigolini, 2014) Hay muchas razones posibles por las que las empresas de LAC (América Latina y el Caribe), por sus siglas en inglés, Latin America and the Caribbean) crecen tan lentamente como lo hacen y una de ellas es la falta de innovación. La entrada no es más que el comienzo de la historia, pues las empresas necesitan innovar continuamente para crecer (o incluso para sobrevivir).

(Zamora Boza C. , 2018) El emprendimiento en Ecuador se lleva a cabo para aprovechar una oportunidad de negocio que permita obtener mayores ingresos, independencia económica y la satisfacción de necesidades. 
Al hacer una comparación regional, el Ecuador se encuentra cerca del promedio de países latinoamericanos con los principales indicadores que miden las aptitudes de los países para desarrollar proyectos productivos. Si bien el ecosistema de emprendimiento en Ecuador se encuentra en transformación, aún es evidente el retraso frente a países como Chile, México, Costa Rica y Colombia en temas de competitividad, innovación y capital social emprendedor.

De acuerdo con la desagregación del índice, las fortalezas de Ecuador están determinadas por las condiciones de la demanda, las políticas y regulaciones pro emprendimiento y las condiciones culturales del país.

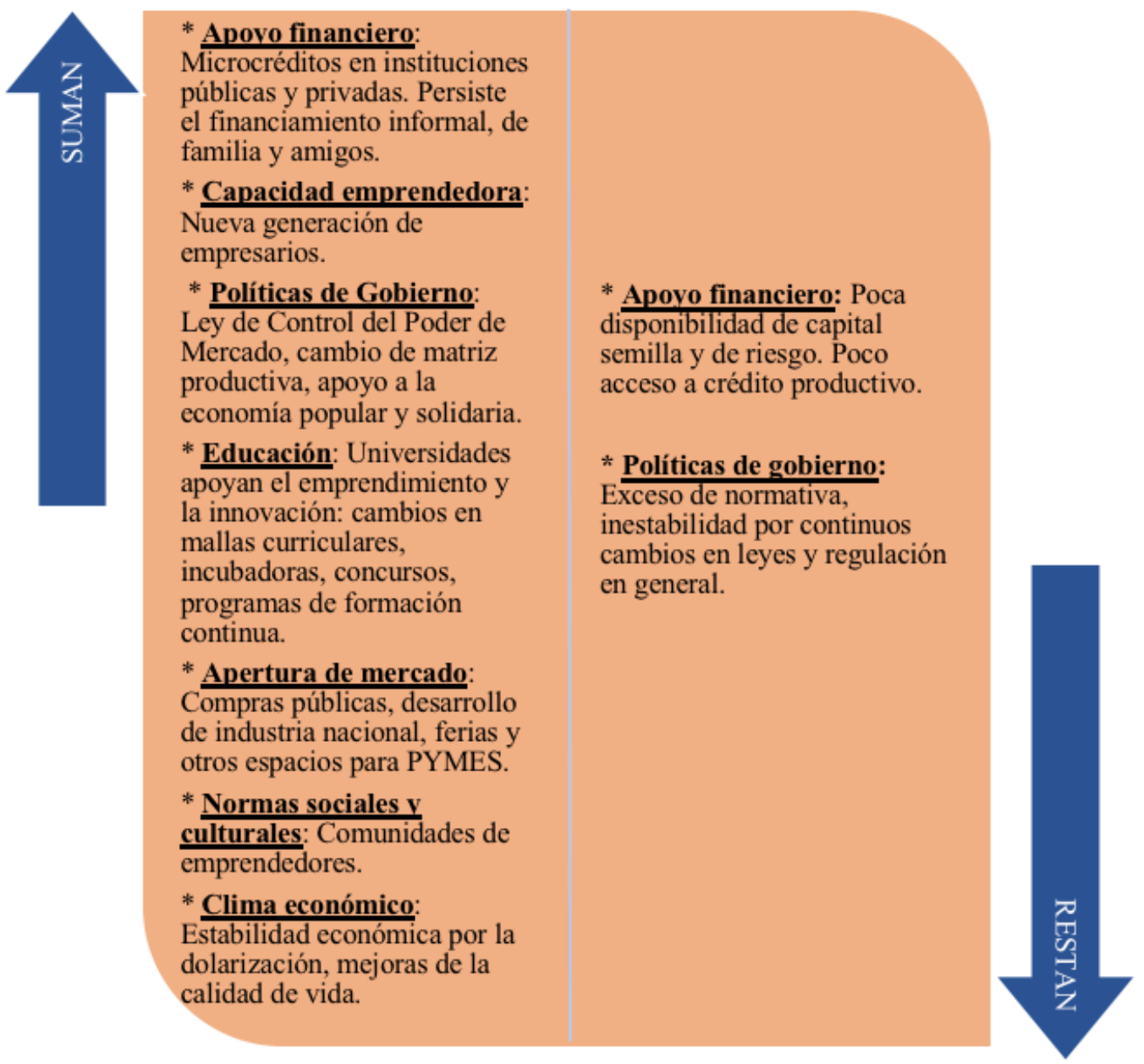

Figura 1. Principales factores que posibilitan y restringen el emprendimiento en Ecuador. Tomado de Zamora-Boza (2018) en Revista Espacios Vol. 39 (No7) Pág. 6 
En los últimos 10 años, la política pública de apoyo al emprendimiento por parte del Gobierno ecuatoriano se ha enfocado en la creación de normativa, instituciones y programas para facilitar la inclusión económica y el desarrollo de actividades productivas en el sector de la economía popular y solidaria (emprendimientos unipersonales, familiares, domésticos, comerciantes minoristas y talleres artesanales, asociaciones productivas, cooperativas financieras y no financieras), así como en los sectores estratégicos (recursos hídricos, hidrocarburos, minería, electricidad y telecomunicaciones).

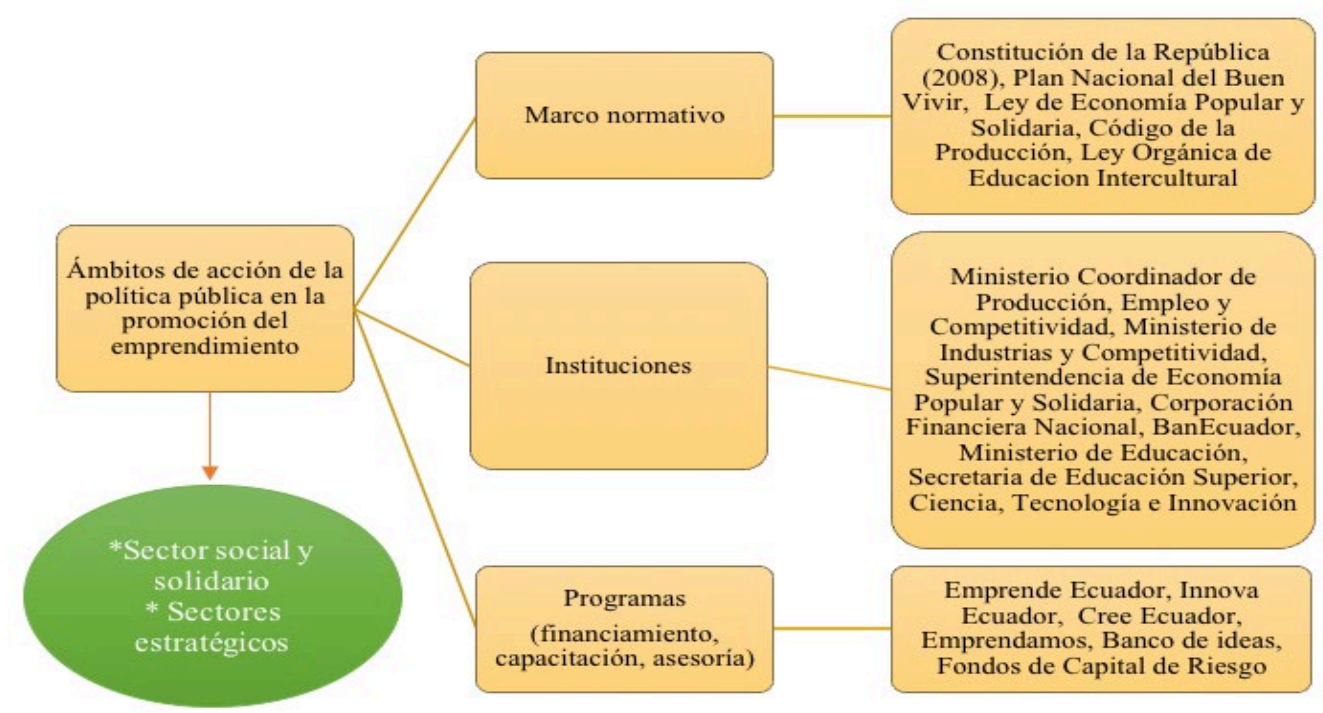

Figura 2. Ámbitos de acción de la política pública en la promoción del emprendimiento. Tomado de Zamora-Boza (2018) en Revista Espacios Vol. 39 ( $\left.N^{o} 07\right)$ Pág. 7

Para (Revista Ekos, 2017) el Ecuador tiene el desafío de desarrollar emprendimientos de calidad enmarcado en los 7 pilares fundamentales: innovación, talento humano, política y marco regulatorio, soporte de mercado, cultura y financiamiento, de éstos el marco regulatorio es débil, los emprendedores ecuatorianos deben enfrentar el riesgo de emprender y el miedo a innovar comparándose con otros países de la región y determinar qué es lo que quieren lograr y hacia dónde quieren llegar.

\section{Grafico No. 4}




\section{Países con TEA más alta en la región}

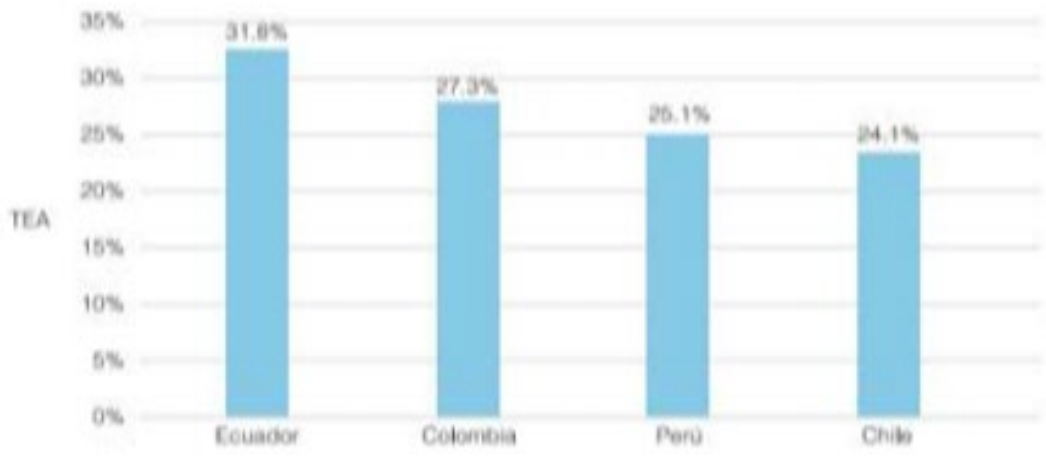

Fuente: Monitor global de emprendimiento, 2016

De acuerdo con el informe del Global Entrepreneurship Monitor (GEM, 2016), la tasa de interés efectiva anual está liderada por Ecuador con el 31,08\% le sigue Colombia con el 27,30\%, Perú con el $25,1 \%$ y Chile con el 24,1\%. Ecuador y Chile muestran reducción en la actividad emprendedora en relación al 2015, mientras que ésta ha aumentado con respecto al año pasado para Colombia y Perú.

\section{Grafico No. 5}

\section{Emprendedores múltiples y el resto de la TEA}

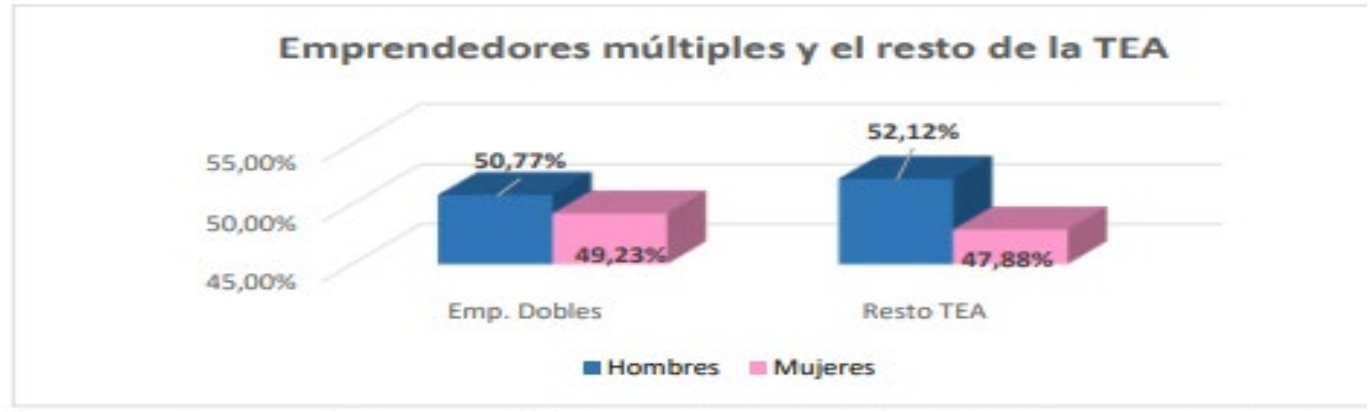

Fuente: Monitor global de emprendimiento, 2016

En este gráfico nos muestra que no hay diferencias sustanciales entre los emprendedores dobles y aquellos que sólo son nacientes o nuevos (el resto de la TEA). Se observa que los hombres presentan mayor participación tanto en los emprendimientos dobles y en aquellos que sólo son nacientes o nuevos (el resto de la TEA). 


\section{Discusión}

La educación financiera es parte importante del desarrollo de los individuos en una sociedad máxime si ésta pretende contribuir al desarrollo socioeconómico de las naciones.

Entender la gestión financiera de su propio negocio es uno de los factores clave para alcanzar el éxito. Un emprendedor que conozca de finanzas para hacer que su dinero invertido produzca, un emprendedor capacitado es un emprendedor de éxito.

Según la organización para la Cooperación y el Desarrollo Económicos (OCDE) entre el 3\% y $10 \%$ de los individuos no tienen una cuenta en el Banco y por consiguiente está aislado del sistema financiero, por lo tanto, la educación financiera es una de las habilidades esenciales que debe desarrollar todo emprendedor.

Según el Global Entrepreneurship Monitor (GEM) la tasa de actividad emprendedora en el Ecuador se sitúa en el 1,8\%, porcentaje inferior al que le correspondería en función de su renta per cápita.

Existe un vínculo estrecho entre la educación financiera y la intención empresarial. En el Ecuador los negocios familiares tienen un impacto en el emprendimiento y la formación empresarial es intrínseca desde la niñez, la curiosidad por emprender viene desde edades muy tempranas.

Si bien la educación financiera por sí sola no significa la solución a la problemática de creación de emprendimientos, también es cierto que, si desde la formación inicial se empieza en la comprensión de conceptos como: finanzas, presupuestos, gestión, gastos, ahorro, impuestos, que se constituyen en la clave para desarrollar la capacidad, cambio de mentalidad y espíritu emprendedor, entonces el país y el mundo conocerán talentos con ideas brillantes, innovadoras y con voluntad para transformar el mundo. (García N., 2018)

\section{Conclusiones}

- Facilitar una buena educación financiera clara, veraz y precisa, hará que las personas inicien con la cultura del ahorro, la búsqueda del financiamiento y la prudencia en el desembolso de gastos, el conocimiento en finanzas influirá en, el éxito de su negocio, mejorará su calidad de vida y gozará de bienestar empresarial y personal.

- En Ecuador la implementación de políticas públicas que norman el desenvolvimiento de estos negocios y que se encuentran tipificados desde la Constitución ha llevado a que las instituciones públicas y privadas estén fomentando la cultura del manejo financiero lo que 
constituye el respaldo a emprendedores que tienen la necesidad de salir adelante y aprovechar las oportunidades del mercado.

- Los emprendimientos generan un número significativo de empleos directos e indirectos remunerados y con permanencia en el tiempo, promueven el desarrollo de las zonas o lugres en donde se establecen sin comprometer el desarrollo sustentable, convirtiéndose en el motor del progreso de los pueblos.

- El apoyo a los emprendedores organizados en el micro y pequeñas empresas ecuatorianas se ve fortalecido con el beneficio para conseguir recursos a tasas más atractivas que las prevalecientes en el mercado, con plazos prudentes para el pago y con la normativa legal flexible creada para apoyar las ideas innovadoras de jóvenes que quieren plasmarlas en realidad.

\section{Referencias Bibliográficas}

Amezcua García, E. L., Arroyo Grant, M. G., \& Espinoza Mejia, F. (2014). Contexto de la Educación Financiera en México. Ciencia administrativa, 24.

Banco del Estado Ecuador. (2015). Taller Educación Financiera. Quito: Ban Ecuador.

Delgado Delgado, D. D., \& Chávez Granizo, G. P. (2018). Las Pymes en el Ecuador y sus fuentes de financiamiento. Eumed.net.

Dominguez Martinez, J. M. (2017). Los programas de Eduación Financiera. Aspectos básicos y referencia al caso español. e-pública.

Espín Oleas, M., Loza Montes, T. M., \& Castillo Armijos, G. J. (2018). Educación Financiera, clave para reducir la pobreza y la desigualdad.

FELABAN. (2016). II Informe de Inclusión Financiera. Bogotá D.C, Colombia. Obtenido de http://www. felaban. net

Fernández de Lis , S., \& Pacheco , L. (2017). Inclusión Financiera en América Latina. XI Foro de Análisis Latinoamericano, Banco de España, 1-30. Obtenido de www. bbvaresearch.com

Fundación Suiza en Bolivia. (2017). Emprendimientos Productivos. Texto Guia del participante. 
García, N. (7 de Marzo de 2018). Educación financiera y el impulso de la educación empresarial para el fomento del emprendimiento. EAE Business Schol.

García, N., Griofoni, A., López, J. C., \& Mejía, D. M. (2013). La educación financiera en América Latina y el Caribe Situación actual y perspectivas. Serie Políticas Públicas y Transformación Productiva $N^{\circ} 12,17$. Obtenido de www.oecd.org

Guerreo Jaimes, R., Villamizar Ramírez, J. M., \& Maestre Delgado, M. (2018). Las finanzas personales desde la educación básica en instituciones de Pamplona. Desarrollo Gerencial, 16.

INEC. (2010). CENSO ECONOMICO . INEC.

Jim Yong Kin. (27 de Febrero de 2015). ¿Poner fin a la pobreza extrema? Comencemos con el acceso a los servicios financieros para todos. Voces peerspectivas del Mundo.

João Carlos Ferraz \& Luma Ramos. (2018). Inclusión financiera para la inserción productiva de las empresas de menor tamaño en América Latina. Cepal.

Lasio, V., Ordeñaña, X., Caicedo, G., Samaniego , A., \& Izquierdo , E. (2017). GLOBAL ENTREPRENEURSHIP MONITOR ECUADOR. Obtenido de http://espae.espol.edu.ec/wp-content/uploads/documentos/GemEcuador2017.pdf

Lederman, D., Messina, J., Pienknagura, S., \& Rigolini, J. (2014). El emprendimiento en America Latina. Muchas empresas y poca innovacion. Washington DC 20433: Creative Commons Attribution CC BY 3.0.

Mejía Anzola, D. M., \& Rodriguez Guzmán, G. (2016). Determinantes socioeconómicos de la Educación Financiera. Evidencia para Bolivia, Colombia, Ecuador y Perú. En D. M. Mejia Anzola, \& G. Rodriguez Guzmán, Determinantes Socioeconómicos de la Educación Financiera. Evidencia para Bolivia. Colombia Ecuador y Perú. Editor CAF.

Moncayo, J., \& Reis, M. (2016). Un análisis inicial del Dinero Electrónico en Ecuador y su impacto en la incluisión financiera. 5.

Moreno Zacarías , H. M., \& Olmos , R. E. (Julio/Diciembre de 2010). Análisis de las características del emprendimiento y liderazgo en los países de Asia y Latinoamérica. 
Revista mexicana de estudios sobre la Cuenca del Pacífico, 4, 1-24. Obtenido de http://www.portesasiapacifico.com.mx

Palacio, G. d. (2017). Medición del conocimiento financiero en los participantes de programas de educación financiera: caso del banco de Lja s.a.

Perspectivas Económicas de America Latina 2017 OCDE/Naciones Unidas/CAF. (2016). CEPAL.

Plan Nacional para el Buen Vivir . (22 de Septiembre de 2017-2021). Plan Nacional para el Buen Vivir . Obtenido de http://www.planificacion.gob.ec

Red de Instituciones Financieras de Desarrollo. (Junio de 2017). Propuesta para la Construcción de una Política Nacional de Inclusión Financiera. Obtenido de http://www.rfr.org.ec

Revista Ekos. (2017). Ecuador tiene la tasa de actividad emprendedora más alta de América Latina. EKOS.

Rios Hamann, X., \& Urbina, M. (2013). Emprendimientos en America Latina: desde la subsistencia hacia la transformacion productiva. Bogota - Colombia: CAF.

Roa, M. J. (2013). Inclusión financiera en América Latina y el Caribe; acceso, uso y calidad. Boletín del CEMLA, 139.

Romero, V. (2017). La importancia de la educación financiera para los emprendedores. Economía y Negocios.

Saavedra Garcia, M. L., \& León Vite, E. L. (2014). Alternativas de financiamiento para la Micro, Pequeña y Mediana Empresa Latinoamericana. Revista Universitaria Ruta Vol. II / 2014, $1-1$.

Salas, E. E., Espinoza, V. d., \& Samaniego, A. (2016). Inclusión Financiera a traves del Crédito de Desarrollo Humano como Herramienta para el Fomento de Capacidades y Emprendimiento. EXPERIENCIAS EN LA MODELACIÓN DE LA TOMA DE DECISIONES EN LA SALUD HUMANA, MEDIO AMBIENTE Y DESARROLLO, 89-103. Obtenido de www.researchgate.net 
Serie Políticas Públicas y transformación productiva No 12. (2013). La eduación financiera en America Latina y el Caribe. Situación actual y perspectivas. CAF.

Villada, F., López Lezama, J. M., \& Muñoz Galeano, N. (2017). El papel de la Educación Financera en la Formación de Profesionales de la Ingeniería. 15.

Yurani Ardila, F., \& Rengifo Ariza, L. E. (2014). Aproximaciones a la Educación Financiera en América Latina. Asociación Nacional de Facultades y Escuelad de Contaduría y Administración.

Zamora Boza, C. S. (2018). La importancia del emprendimiento en la economía: Caso Ecuador. Revista Espacios.

Zúñiga Santillán, X. L., Espinoza Toalombo, R. A., Campos Rocafuerte, ,. H., Tapia Núñez, ,. D., \& Muñoz Bernal, ,. M. (2016). UNA MIRADA A LA GLOBALIZACIÓN: PYMES ECUATORIANAS. OBSERVATORIO DE LA ECONOMIA LATINOAMERICANA, 17. 